TRANSACTIONS OF THE

AMERICAN MATHEMATICAL SOCIETY

Volume 363, Number 3, March 2011, Pages 1265-1285

S 0002-9947(2010)05316-4

Article electronically published on October 22, 2010

\title{
ON NONPOSITIVELY CURVED COMPACT RIEMANNIAN MANIFOLDS WITH DEGENERATE RICCI TENSOR
}

\author{
DINCER GULER AND FANGYANG ZHENG
}

\begin{abstract}
In this article, we prove that if the Ricci tensor of a compact nonpositively curved manifold $M^{n}$ is nowhere negative definite, then it admits local flat factors. To be more precise, let $U \subseteq M$ be the open subset where the Ricci tensor has maximum rank $r$. Then for any connected component $U_{a}$ of $U$, its universal cover $\widetilde{U_{a}}$ is isometric to $\mathbf{R}^{n-r} \times N_{a}^{r}$, where $N_{a}^{r}$ is a nonpositively curved manifold with negative Ricci curvature.

In particular, if $M^{n}$ is compact, nonpositively curved without Euclidean de Rham factor, and both the manifold and the metric are real analytic, then its Ricci tensor must be negative definite somewhere.
\end{abstract}

\section{Contents}

1. Introduction 1265

2. Cartan-Hadamard manifolds with degenerate Ricci $\quad 1269$

2.1. The $R$-splits $\quad 1270$

2.2. Further restrictions 1274

3. The proof of Theorem $1 \quad 1278$

3.1. The type J case $\quad 1279$

3.2. The type $\mathrm{N}$ case $\quad 1280$

3.3. The general case $\quad 1282$

Acknowledgement $\quad 1284$

\begin{tabular}{ll} 
References & 1284 \\
\hline
\end{tabular}

\section{INTRODUCTION}

In the study of nonpositively curved manifolds, it is often very important to distinguish between the negatively curved case and the nonpositively curved case. Because in the negatively curved situation a manifold usually possesses certain hyperbolicity, while in the nonpositively curved but not the negatively curved case, some rigidity phenomena often occur.

Received by the editors September 19, 2008.

2000 Mathematics Subject Classification. Primary 53C20; Secondary 53C12.

Key words and phrases. Nonpositive sectional curvature, Euclidean de Rham factor, Ricci tensor, Ricci rank, totally geodesic foliation, conullity operator.

This research was partially supported by an NSF Grant, the Ohio State University, the IMS of CUHK and the CMS of Zhejiang University.

(C)2010 American Mathematical Society Reverts to public domain 28 years from publication 
Of course one natural way for a nonpositively curved manifold $M^{n}$ to be not negatively curved occurs when it has degenerate Ricci tensor; that is, when the maximum rank of the Ricci tensor is less than its dimension. In this case, at each point in $M^{n}$ there will be some direction in which the Ricci curvature is zero; thus there are many zero sectional curvatures at any point.

A trivial way for this to happen occurs when $M^{n}$ (or rather, its universal covering space $\widetilde{M}^{n}$ ) has a nontrivial Euclidean de Rham factor. The beautiful Eberlein theory ([CE], [E1]-E5]; see also [EHS] and the references therein) provides a complete answer to this when $M^{n}$ is compact. In this case, there always exists a finite cover $M^{\prime}$ of $M^{n}$, which is a metric fiber bundle over a compact nonpositively curved manifold $N$ without Euclidean de Rham factor, while the fiber is a flat torus (whose dimension is the dimension of the Euclidean de Rham factor of $\widetilde{M}^{n}$ ).

So we may just consider a compact, nonpositively curved Riemannian manifold $M^{n}$ (whose universal cover is) without Euclidean de Rham factor. Denote by $r$ the maximum of the rank of its Ricci tensor. We will call it the Ricci rank of $M$. We say that $M^{n}$ has degenerate Ricci if $r<n$.

Note that even when $M^{n}$ is without Euclidean de Rham factor, it still could happen that $r<n$. For instance, take a punctured torus $Y$ and equip it with a nonpositively curved metric such that a neighborhood of the boundary is isometric to $[0,1] \times S^{1}$. Take two copies of $Y \times S^{1}$ and glue them along the boundary torus, but with the $S^{1}$ factors switched. The resulting space is a closed 3-manifold $M^{3}$ with nonpositive sectional curvature, with Ricci rank 2, and without Euclidean de Rham factor. (This example is often called Gromov's example, and we first learned it from X. Rong.)

More generally, any graph manifold $M^{3}$ which admits a nonpositively curved metric (and without flat de Rham factor) would have Ricci rank equal to 2.

It is natural to propose the following

Conjecture A. Let $M^{n}$ be a compact Riemannian manifold with nonpositive sectional curvature, with degenerate Ricci tensor, and without Euclidean de Rham factor. Then it must be a generalized graph manifold in the sense of Cao-Cheeger-Rong $\mathrm{CCR}$.

We refer the readers to the recent work [CCR] for the precise definition of their generalized graph manifold. In it, Cao, Cheeger and Rong were able to show that $M^{n}$ above is a generalized graph manifold if it is in the collapsing case, namely, if the sectional curvature $K$ of $M^{n}$ is between -1 and 0 , and its injectivity radius at each point is less than a universal constant $\epsilon(n)$, which is basically the Margulis constant divided by the Bieberbach constant. In the collapsing case, one always has degenerate Ricci ( $[\mathrm{CCR}]$ ), but one can see from the above example that the converse is not always true, since the circles there need not have a small radius.

In this paper, we will use a totally different approach to prove the following result, which provides a partial answer to Conjecture A.

Theorem 1. Let $M^{n}$ be a complete Riemannian manifold with nonpositive and bounded sectional curvature, with finite volume, and with Ricci rank $r<n$. Denote by $U \subseteq M^{n}$ the open subset where the Ricci tensor has rank $r$. Then for any connected component $U_{a}$ of $U$, its universal cover $\widetilde{U_{a}}$ is isometric to $\mathbf{R}^{n-r} \times N_{a}^{r}$, where $N_{a}^{r}$ is a nonpositively curved manifold with negative Ricci curvature. 
Theorem 1 can be regarded as a test case for Conjecture A. Note that to confirm Conjecture A, however, one still has to at least show that the leaves $\mathbf{R}^{n-r} \times\{x\}$ for any $x \in N_{a}^{r}$ actually have compact images in $M^{n}$. This will give the local torus actions on (the most curved part $U$ of) $M^{n}$.

Although at this point we are still far from being able to solve the conjecture, we do believe that the key here might be buried somewhere in the beautiful and deep theory of Eberlein on Euclidean de Rham factors and the rigidity phenomenon (see the aforementioned references). We hope that the ideas and techniques there could be further exploited to help in 'closing up' the leaves of the Ricci kernel foliation $\mathcal{L}$. As an example to illustrate the far reaching influence of the Eberlein theory, sometimes to seemingly quite different circumstances, we would like to just mention the recent work WZ1, which studied uniformization theory for compact Kähler manifolds with nonpositive bisectional curvature. Despite the obvious difference in the situations and proofing methods, the Eberlein theory served as an important 'guiding light' throughout that work.

Remark. As one can see from the proof of Theorem 1, the boundedness assumption on the curvature is only needed for each connected component of the open subset $U$, instead of the entire manifold, and no uniform bound is required.

This remark was suggested by the referee of this paper, for the reason that a general noncompact complete graph manifold with nonpositive sectional curvature may not have a bounded curvature but could meet the weaker restriction on curvature.

In the special case when $M^{n}$ in Theorem 1 is also real analytic (this means, both the manifold and the metric are real analytic), since local splitting always implies global splitting in the analytic case, we know that $\widetilde{M}$ will have nontrivial Euclidean de Rham factor when $r<n$. In other words, we have

Corollary 2. Let $M^{n}$ be a complete Riemannian manifold with finite volume and with bounded, nonpositive sectional curvature. Assume that it is without Euclidean de Rham factor, and both the manifold and the metric are real analytic. Then the Ricci tensor is negative definite somewhere.

The referee of this paper also suggested that we highlight the following special case of Theorem 1, for the reason that, in the study of rigidity, compact manifolds are mostly studied and understood.

Corollary 2a. Let $M^{n}$ be a compact Riemannian manifold with nonpositive sectional curvature and with Ricci rank $r<n$. Denote by $U \subseteq M^{n}$ the open subset where the Ricci tensor has rank $r$. Then for any connected component $U_{a}$ of $U$, its universal cover $\widetilde{U_{a}}$ is isometric to $\mathbf{R}^{n-r} \times N_{a}^{r}$, where $N_{a}^{r}$ is a nonpositively curved manifold with negative Ricci curvature.

Our approach towards Theorem 1 is to analyze the structure of Cartan-Hadamard manifolds with degenerate Ricci. There are many examples of Cartan-Hadamard manifolds with degenerate Ricci but without Euclidean de Rham factor, as observed in [GZ], which borrowed some examples from [WZ]. However, such manifolds tend to have very restrictive structures. By a careful analysis on the curvature equations (which is a continuation of the line of work in GZ]), we obtain a structural result (Theorems 4-8 in §2) for such manifolds. It turns out that such a manifold admits local splitting into irreducible blocks and that each nonflat block with degenerate Ricci is either of type $J$ or of type $N$. In both types the geometric behavior of the 
conullity operators can be precisely described. The results in $\S 2$ should also be of some independent interest, and perhaps should have a version in the CAT(0) and other generalized situations.

In $\S 3$ we show that neither type $\mathrm{J}$ nor type $\mathrm{N}$ cases can survive the action of a large isometry group such as a lattice. This together with the splitting result in $\S 2$ would lead to a proof of Theorem 1.

Before we end this section, let us make two more remarks here. First, under the real analyticity assumption, to what extent can Corollary 2 be strengthened? For instance, the following was raised by Viktor Schroeder ([S5]):

Conjecture B (Schroeder). Let $M^{n}$ be a compact, nonpositively curved, real analytic Riemannian manifold. If an open dense subset $U \subset M^{n}$ is (real analytically) foliated by totally geodesic flats of dimension at least 2, then (the universal cover of) $M^{n}$ must contain a nontrivial Euclidean de Rham factor.

In this direction, the papers $[\underline{\mathrm{S} 1}-\underline{\mathrm{S} 4}$ and $[\mathrm{BS}]$ provided the best known results. In $\overline{B S}$, Bangert and Schroeder proved that if the universal cover contains a totally geodesic flat of dimension at least 2 , then $M^{n}$ will contain a totally geodesic flat torus. They achieved this through studying the (real analytic) dynamics of the geodesic flow. Hopefully their techniques can be borrowed and further developed to provide an answer to this problem, as well as towards the general picture of the dynamics of the geodesic flow for nonpositively curved real analytic manifolds.

One may also drop the analyticity assumption in Conjecture B and ask a slightly more general question: if $M^{n}$ is compact, nonpositively curved, and $\mathcal{F}$ is a totally geodesic foliation in an open subset $U \subseteq M^{n}$ whose leaves are complete flats with dimension at least 2 , then must the universal cover of each connected component of $U$ be isometric to a product with a flat factor?

At this point an affirmative answer to Conjecture B or the above generalization still seems to be distant, because one is dealing with an arbitrary foliation (of totally geodesic flats). The specialty of the foliation in Theorem 1 is that it is the kernel of the Ricci, thus closely tied to the geometry of the manifold, and what we are doing here is carefully examining its leaf structure and its transversal geometric and topological behavior.

The second remark is that there are several questions that are naturally related to Conjecture A. For instance, we will again mention the following conjecture raised in [GZ], which says that the Ricci rank is a homotopy invariant:

Conjecture C. If $M^{n}, N^{n}$ are two compact, nonpositively curved Riemannian manifolds and are homotopy equivalent to each other, then their Ricci rank must be equal.

Eberlein theory on Euclidean de Rham factors says that the dimension of their Euclidean de Rham factors are the same, because they both equal the rank of the Clifford group of the (same) fundamental group.

Some closely related questions were raised and studied in $[\mathrm{CCR}$ and its sequels to come. Perhaps the harmonic map technique will be useful in dealing with Conjecture C. See for instance [JY] for the global product case.

The main result of this article was announced in [Z1]. 


\section{Cartan-Hadamard manifolds With Degenerate Ricci}

In this section, we study the structure of a Cartan-Hadamard manifold $M^{n}$ with degenerate Ricci curvature. The main result would be the structural theorems (Theorems 4-8). The proofs are along the line of [Z] and [GZ; in the latter an inequality about the Ricci rank was established.

Let us begin by fixing some notation. Throughout this section, $M^{n}$ will be a Cartan-Hadamard manifold with degenerate Ricci. That is, $M^{n}$ is a complete, simply-connected Riemannian manifold with nonpositive sectional curvature, and its Ricci rank $r$, namely the maximum rank of the Ricci tensor, satisfies $0<r<n$.

Let $U \subseteq M^{n}$ be the open subset where the Ricci tensor has rank $r$, and let $\mathcal{L}$ be the distribution in $U$ given by the kernel of the Ricci tensor. The nonpositivity of the sectional curvature implies that $\mathcal{L}$ coincides with the kernel foliation of the Riemannian curvature tensor $R$. Thus $\mathcal{L}$ is a totally geodesic foliation whose leaves are complete, totally geodesic flat submanifolds in $M^{n}$. By an abuse of notation, we will use the symbol $\mathcal{L}$ to denote both the distribution and the foliation. Note that the completeness of the leaves is due to a result by Ferus $[\mathrm{F}$. From now on, we will call $\mathcal{L}$ the Ricci kernel foliation of $M^{n}$.

Denote by $\mathcal{L}^{\perp}$ the distribution in $U$ that is the orthogonal complement of $\mathcal{L}$. Recall that the conullity operators $C_{T}$ are defined by

$$
C_{T}(X)=-\left(\nabla_{X} T\right)^{\perp}
$$

where $T$ and $X$ are vector fields with $T \in \mathcal{L}$ and $X \in \mathcal{L}^{\perp}$. Here $Y^{\perp}$ stands for the $\mathcal{L}^{\perp}$ component of $Y$. These operators are well-defined tensor fields, and they satisfy the Riccati type equations

$$
\nabla_{S} C_{T}=C_{S} C_{T}
$$

as well as the so-called curvature equations

$$
R\left(X, Y, C_{T} Z, W\right)+R\left(Y, Z, C_{T} X, W\right)+R\left(Z, X, C_{T} Y, W\right)=0
$$

for any $T, S \in \mathcal{L}$ with $\nabla_{S} T=0$ and any $X, Y, Z, W \in \mathcal{L}^{\perp}$. The Riccati equations, together with the completeness of the leaves, force any $C_{T}$ to have no nonzero real eigenvalues $([\mathrm{DR}])$.

We refer the readers to the papers $[\mathrm{F}, \mathrm{DR}]$ and $\mathrm{GZ}]$ for the references on these claims. The algebraic analysis of GZ, using the curvature conditions and the curvature equations, provided further restrictions on those conullity operators. Let us denote by $\mathcal{C}$ the real vector space formed by $C_{T}$ for all $T \in \mathcal{L}_{x}$ at a point $x \in U$.

Definition. For any $x \in U$, we will say that the curvature tensor $R$ splits at $x$ if there is some nontrivial decomposition $\mathcal{L}_{x}^{\perp}=V_{1} \oplus V_{2}$ such that for any vectors $X, Y, Z, W \in V_{1} \cup V_{2}, R(X, Y, Z, W)=0$ unless all four vectors are in $V_{1}$ or all four are in $V_{2}$. Such a decomposition will be called an $R$-split.

Note that in this case each $C_{T}$ will preserve the decomposition; that is, $C_{T}\left(V_{i}\right) \subseteq$ $V_{i}$ for $i=1,2$ (see Lemma 1 of [GZ]).

The analysis on the curvature equations in GZ says the following:

Theorem 3 (GZ). If $R$ does not split at $x \in U$, then at $x$ either all $C_{T}$ are nilpotent matrices and the product of any two of them is zero, or all $C_{T}$ are in the form $a I+b D$, where $I$ is the identity matrix and $D^{2}=-I$. Note that in the latter case, the Ricci rank $r$ (which is the dimension of $\mathcal{L}_{x}^{\perp}$ ) is necessarily an even integer. 
For convenience, we will call the first case type $N$ ( $\mathrm{N}$ for nilpotent) and the second case type $J$ ( $\mathrm{J}$ for an almost complex structure).

Based on this, [GZ proved the inequality $r \geq n-r-s$, or equivalently, $2 r-1 \geq n-s$. Here $s$ is the core number of $M^{n}$, defined as follows.

For any $x \in U$, denote by $\mathcal{K}_{x}$ the subspace of $\mathcal{L}_{x}$ consisting of all $T$ with $C_{T}=0$. The core number $s$ is just the minimum dimension of $\mathcal{K}_{x}$ for all $x \in U$. Write $U^{\prime} \subseteq U$ for the open subset where $\mathcal{K}_{x}$ has constant dimension $s$. In $U^{\prime}, \mathcal{K}$ becomes a distribution contained in $\mathcal{L}$, and clearly it is a totally geodesic foliation as well. Let us write $\mathcal{L}=\mathcal{K} \oplus \mathcal{L}^{\prime}$ in $U^{\prime}$ for the orthogonal decomposition.

Note that just like $U$, the set $U^{\prime}$ is $\mathcal{L}$-complete in the sense that, if $x \in U^{\prime}$, then the entire leaf $\mathcal{L}_{x} \subseteq U^{\prime}$. Also, the Euclidean de Rham factor of $M^{n}$, if it exists, goes into this $\mathcal{K}$. But even when $M^{n}$ is irreducible, $\mathcal{K}$ can still be positive dimensional as shown by Example 1 in $\S 1$ of [GZ], which was borrowed from [WZ].

In rough terms, the conullity operators measure the 'twisting' and 'turning' of the leaves of $\mathcal{L}$. The leaves of $\mathcal{K}$ move parallel within $\mathcal{L}$ and do not 'twist' in the $\mathcal{L}^{\perp}$ directions by definition (i.e., $\left\langle\nabla_{\mathcal{L}^{\perp}} \mathcal{K}, \mathcal{L}^{\perp}\right\rangle=0$ ), but they can be 'turning' and thus not parallel to each other (that is, $\left\langle\nabla_{\mathcal{L}^{\perp}} \mathcal{K}, \mathcal{L}^{\prime}\right\rangle$ may not be zero). The core number $s$ provides a good measurement to the flatness of the manifold $M^{n}$.

Our goal in this section is to establish a local product structure near a generic point in any Cartan-Hadamard manifold $M^{n}$ which has degenerate Ricci and whose $\mathcal{L}^{\perp}$ admits an $R$-split everywhere.

2.1. The $R$-splits. Let us fix an arbitrary point $x \in U$ and consider the vector space $V=\mathcal{L}_{x}^{\perp}$. Let $V=V_{1} \oplus V_{2} \oplus \cdots \oplus V_{k}$ be an $R$-split into irreducible blocks, that is, each $V_{i}$ does not admit a further $R$-split. We continue our discussion with a series of claims.

Claim 1. At any $x \in U$, the $R$-split of $V$ into irreducible subspaces is unique.

Proof. Suppose that $V=P \oplus Q$ is an $R$-split. We want to show that

$$
P=\bigcup_{i \in A} V_{i}, \quad Q=\bigcup_{i \in B} V_{i},
$$

where $\{1,2, \ldots, k\}$ is the disjoint union of $A$ and $B$. To see that, let $\pi_{i}: V \rightarrow V_{i}$ be the projection map, $P_{i}=\pi_{i}(P), Q_{i}=\pi_{i}(Q)$. For any $X \in P$ and any $Y \in Q$, we have

$$
0=K(X, Y)=\sum_{i=1}^{k} K\left(X_{i}, Y_{i}\right)
$$

where $X_{i}=\pi_{i}(X), Y_{i}=\pi_{i}(Y)$, and $K(X, Y)=R(X, Y, Y, X)$. So by the nonpositivity of the sectional curvature, we get $K\left(X_{i}, Y_{i}\right)=0$ for each $i$.

Since $V=P \oplus Q$, we have $V_{i}=P_{i}+Q_{i}$. If $Z \in P_{i} \cap Q_{i}$, then $K(Z, X)=0$ for any $X$ in $P_{i}$ or $Q_{i}$, hence for any $X \in V_{i}$. So $K(Z, X)=0$ for any $X \in V$, and by the negativity of the Ricci tensor on $V$, we know that $Z=0$. That is, $V_{i}=P_{i} \oplus Q_{i}$ for each $i$. By the irreducibility of $V_{i}$, either $P_{i}=0$ or $Q_{i}=0$.

Write $P^{\prime}=\bigoplus_{i} P_{i}$ and $Q^{\prime}=\bigoplus_{i} Q_{i}$. Then $P^{\prime} \supseteq P$ and $Q^{\prime} \supseteq Q$. So based on the fact

$$
V=\bigoplus_{i} V_{i}=P^{\prime} \oplus Q^{\prime}=P \oplus Q,
$$

we know that $P=P^{\prime}$ and $Q=Q^{\prime}$ are both the direct sum of some $V_{i}$ 's. This completes the proof of Claim 1. 
Claim 2. The $R$-split pattern of $\mathcal{L}^{\perp}$ remains constant along each leaf of $\mathcal{L}$.

Proof. Let $\gamma(t)$ be a geodesic contained in a leaf of $\mathcal{L}$, and write $x=\gamma(0)$. Suppose $\mathcal{L}_{x}^{\perp}=V_{1} \oplus V_{2}$ is an $R$-split. Extend $V_{1}$ and $V_{2}$ parallel along $\gamma$. Then we claim that they give an $R$-split at any $\gamma(t)$ as well. To see this, let $X, Y, Z, W$ be any parallel vectors fields along $\gamma(t)$, where each belongs to $V_{1} \cup V_{2}$ but not all four are in the same $V_{1}$ or $V_{2}$. The second Bianchi identity gives

$$
\nabla_{T} R_{X Y Z W}=R_{C_{T}(X), Y Z W}+R_{X, C_{T}(Y), Z W}
$$

where $T=\gamma^{\prime}(t)$. Since $\nabla_{T} C_{T}=\left(C_{T}\right)^{2}$, by $C_{T}\left(V_{i}\right) \subseteq V_{i}(i=1,2)$ at $t=0$ we know the same holds true at any $\gamma(t)$. Thus the above identity on ceurvature implies that $V_{1} \oplus V_{2}$ also gives an $R$ split at any $\gamma(t)$.

This means that the splitting pattern of $\mathcal{L}^{\perp}$ remains constant along each leaf of $\mathcal{L}$.

Claim 3. Suppose $\left\{x_{\alpha}\right\}$ is a sequence of points in $U$ approaching a point $x \in U$ such that for each $\alpha, \mathcal{L}_{x_{\alpha}}^{\perp}=V_{1}\left(x_{\alpha}\right) \oplus \cdots \oplus V_{l}\left(x_{\alpha}\right)$ is an $R$-split at $x_{\alpha}$ and $\lim _{\alpha \rightarrow \infty} V_{i}\left(x_{\alpha}\right)=$ $V_{i}$ for each $1 \leq i \leq l$. Then

$$
\mathcal{L}_{x}^{\perp}=V_{1} \oplus \cdots \oplus V_{l}
$$

is a direct sum and is an $R$-split.

Proof. We just need to show that the above sum of the limiting subspaces is a direct sum. For this it suffices to show that $V_{i} \cap W_{i}=0$ for each $i$, where $W_{i}=\sum_{j \neq i} V_{j}$. Define $W_{i}\left(x_{\alpha}\right)$ similarly. From the definition of an $R$-split, we know that

$$
R(X, *, *, Y)=0
$$

for any $X \in V_{i}\left(x_{\alpha}\right)$ and any $Y \in W_{i}\left(x_{\alpha}\right)$. By letting $\alpha \rightarrow \infty$, we know that the same is true at $x$. So if $X \in V_{i} \cap W_{i}$, the Ricci curvature in $X$ would be zero, contradicting the fact that the Ricci tensor is negative definite in $\mathcal{L}^{\perp}$. This proves the claim.

Now let us write $k(x)$ for the number of irreducible $R$-blocks at $x \in U$, and let $k$ be its minimum for all $x \in U$. Denote by $U^{*}$ the open subset of $U$, where $k(x)=k$. By the compactness of the Grassmannians and Claims 1 and 3, we have distributions $\mathcal{V}_{1}, \ldots, \mathcal{V}_{k}$ in $U^{*}$ such that

$$
\mathcal{L}^{\perp}=\mathcal{V}_{1} \oplus \cdots \oplus \mathcal{V}_{k}
$$

and it is the irreducible $R$-split at each $x \in U^{*}$. By Claim 2, we know that $U^{*}$ is $\mathcal{L}$-complete; namely, if $x \in U^{*}$, then the entire leaf $\mathcal{L}_{x} \subseteq U^{*}$. Also, each distribution $\mathcal{V}_{i}$ is parallel along any leaf of $\mathcal{L}$.

By its construction, we also know that the subset $U^{*}$ is invariant under the isometry group of $M^{n}$; that is, for any $f \in I$ so $(M), f\left(U^{*}\right)=U^{*}$.

Next, we want to use these $\mathcal{V}_{i}$ to generate a local product structure on (an open, invariant, and $\mathcal{L}$-complete subset of) $U^{*}$, so that each (nonflat) factor does not admit an $R$-split anymore.

Claim 4. For each $1 \leq i \leq k, \nabla_{\left(\mathcal{V}_{i} \oplus \mathcal{L}\right)}\left(\mathcal{V}_{i} \oplus \mathcal{L}\right) \subseteq\left(\mathcal{V}_{i} \oplus \mathcal{L}\right)$.

Proof. Note that there is nothing to prove if $k=1$, so we may assume $k \geq 2$. Without loss of generality, we may assume $i=1$ and $k=2$ (just write the direct sum of the other $k-1$ factors as $\mathcal{V}_{2}$ ). So we have a decomposition

$$
T M=\mathcal{L} \oplus \mathcal{V}_{1} \oplus \mathcal{V}_{2}
$$


Let $\left\{e_{0}, e_{\alpha}, e_{i}\right\}$ be a local tangent frame near $x$ with $e_{0}$ 's in $\mathcal{L}, e_{\alpha}$ 's in $\mathcal{V}_{1}$ and $e_{i}$ 's in $\mathcal{V}_{2}$. Let $g, \theta$ and $\Theta$ be the $n \times n$ matrices of the metric, connection and curvature under the frame. Also write $\Omega=\Theta g$ and $\xi=g^{-1} \theta g-g^{-1} d g$. We have

$$
R_{0 * * *}=0, \quad R_{i \alpha * *}=0 .
$$

Therefore, $\Omega_{i \alpha}=\Omega_{\alpha i}=0$. On the other hand, the second Bianchi identity leads to $d \Omega=\theta \Omega-\Omega \xi$. Thus

$$
0=d \Omega_{\alpha i}=\sum_{j} \theta_{\alpha j} \Omega_{j i}-\sum_{\beta} \Omega_{\alpha \beta} \xi_{\beta i} .
$$

Hence, $\sum_{j} \theta_{\alpha j} \Omega_{j i}=0, \sum_{\beta} \Omega_{\alpha \beta} \xi_{\beta i}=0$. From this and the fact that the Ricci tensor is negative definite in $\mathcal{L}^{\perp}$, we get

$$
\theta_{\alpha j}\left(e_{0}\right)=0, \quad \theta_{\alpha j}\left(e_{\beta}\right)=0
$$

for any $\alpha, j$, and any $e_{\beta}$ and $e_{0}$. This proves Claim 4 .

Claim 5. The decomposition $\mathcal{L}^{\perp}=\mathcal{V}_{1} \oplus \cdots \oplus \mathcal{V}_{k}$ is orthogonal.

Proof. To see this, let us denote that

$$
\mathcal{W}_{i}=\operatorname{span}\left\{R(X, Y) Z \mid X, Y, Z \in \mathcal{V}_{i}\right\} .
$$

By Claim 4, we know that $\mathcal{W}_{i} \subseteq \mathcal{L} \oplus \mathcal{V}_{i}$. On the other hand, for any $X, Y, Z \in \mathcal{V}_{i}$, $R(X, Y, Z, W)=0$ unless $W \in \mathcal{V}_{i}$ as well, so

$$
\mathcal{W}_{i} \subseteq\left(\bigoplus_{j \neq i} \mathcal{V}_{j} \oplus \mathcal{L}\right)^{\perp}=\left(\bigoplus_{j \neq i} \mathcal{V}_{j}\right)^{\perp} \cap \mathcal{L}^{\perp}
$$

Thus $\mathcal{W}_{i} \subseteq \mathcal{V}_{i}$ for each $i$. Now let $\mathcal{V}_{i}=\mathcal{W}_{i} \oplus \mathcal{P}_{i}$ be the orthogonal decomposition. For any $X, Y, Z$ and $W$ in $\mathcal{W}_{i} \cup \mathcal{P}_{i}$, we have

$$
R(X, Y, Z, W)=0
$$

if any one of the four vectors is in $\mathcal{P}_{i} \perp \mathcal{W}_{i}$. So this gives an $R$-split. By the irreducibility of $\mathcal{V}_{i}$, we get $\mathcal{W}_{i}=\mathcal{V}_{i}$. (Note that the other possibility, namely $\mathcal{W}_{i}=0$, would contradict the fact that the Ricci tensor needs to be negative definite on $\mathcal{V}_{i}$.) This means $\mathcal{V}_{i} \subseteq\left(\bigoplus_{j \neq i} \mathcal{V}_{j}\right)^{\perp}$, so $\mathcal{V}_{i} \perp \mathcal{V}_{j}$ for any $i \neq j$, and Claim 5 is proved.

Because of Claim 5, in the proof of Claim 4 we may choose our tangent frame to be an orthonormal one, so $g=I, \Omega=\Theta$ and $\xi=\theta$. By the condition

$$
\sum_{\beta} \Omega_{\alpha \beta} \xi_{\beta i}=0 \quad \forall \alpha, \forall i
$$

we conclude $\theta_{\alpha i}\left(e_{j}\right)=0$, hence $\theta_{\alpha i}=0$. Therefore we have for each $i$ that

$$
\nabla_{*} \mathcal{V}_{i} \subseteq \mathcal{V}_{i} \oplus \mathcal{L}
$$

It is also not hard to see that $\theta_{\alpha 0}\left(e_{j}\right)=\theta_{\alpha 0}\left(e_{0^{\prime}}\right)=0$; that is,

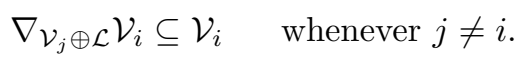

Now we are ready to construct the local product splitting. Assume that $R$ does split everywhere in $U$; that is, assume $k \geq 2$. We have an orthogonal decomposition

$$
T M=\mathcal{L} \oplus \mathcal{V}_{1} \oplus \cdots \oplus \mathcal{V}_{k}
$$


in the open subset $U^{*}$. Also,

$$
\nabla_{\mathcal{V}_{i}^{\perp}} \mathcal{V}_{i} \subseteq \mathcal{V}_{i}, \quad \nabla_{\mathcal{V}_{i} \oplus \mathcal{L}}\left(\mathcal{V}_{i} \oplus \mathcal{L}\right) \subseteq \mathcal{V}_{i} \oplus \mathcal{L}
$$

Following a trick in FZ1, at any $x \in U^{*}$ let us define subspaces of the tangent space at $x$ by first letting $\mathcal{V}_{i}^{0}=\mathcal{V}_{i}$, and then inductively for any $m \geq 0$,

$$
\mathcal{V}_{i}^{m+1}=\mathcal{V}_{i}^{m}+\operatorname{span}\left\{\nabla_{X} Y \mid X \in \mathcal{V}_{i}, Y \in \mathcal{V}_{i}^{m}\right\} .
$$

Clearly we have $\mathcal{V}_{i}^{m} \subseteq \mathcal{V}_{i} \oplus \mathcal{L}$ for all $m$. It is also not hard to show by induction that

$$
\nabla_{\mathcal{V}_{i}^{\perp}} \mathcal{V}_{i}^{m} \subseteq \mathcal{V}_{i}^{m}, \quad \mathcal{V}_{i}^{m} \perp \mathcal{V}_{j}^{l}
$$

for any $i \neq j$ and any $m, l \geq 0$. Now let us denote by $\mathcal{V}_{i}^{\infty}$ the union of $\mathcal{V}_{i}^{m}$ for all $m \geq 0$. Denote by $U^{* *}$ the nonempty open subset of $U^{*}$ where each $\mathcal{V}_{i}^{\infty}$ reaches its maximum dimension, then within $U^{* *}$ each $\mathcal{V}_{i}^{\infty}$ becomes a distribution, and by the properties of $\mathcal{V}_{i}^{m}$ they satisfy

$$
\nabla_{*} \mathcal{V}_{i}^{\infty} \subseteq \mathcal{V}_{i}^{\infty}, \quad \mathcal{V}_{i}^{\infty} \subseteq \mathcal{V}_{i} \oplus \mathcal{L}
$$

for each $i$. The first condition says that the distribution $\mathcal{V}_{i}^{\infty}$ is parallel, and the second says that it is proper in $T M$ since $k \geq 2$. Thus the open subset $U^{* *}$ will be a local product, corresponding to the distribution decomposition in $U^{* *}$

$$
T M=\mathcal{V}_{1}^{\infty} \oplus \cdots \oplus \mathcal{V}_{k}^{\infty} \oplus \mathcal{L}^{0},
$$

where the last factor is defined to be the orthogonal complement of the sum of the previous $k$ factors, with dimension $q \geq 0$ (which could be 0 ).

Here and after, 'local product structure' on $U^{* *}$ means that, for each connected component $U_{a}^{* *}$ of $U^{* *}$, its universal cover $\widetilde{U_{a}^{* *}}$ is isometric to a product space

$$
Y_{1 a} \times \cdots \times Y_{k a} \times \mathbf{R}^{q}
$$

where $q \geq 0$ is the dimension of $\mathcal{L}^{0}$ above, and each $Y_{i a}$ is a nonpositively curved Riemannian manifold foliated by its Ricci kernel foliation $\mathcal{L}_{i a}$ whose leaves are complete totally geodesic flats, and in the transversal directions, the Ricci is negative definite and there is no $R$-split. Note that for some factors this foliation could be trivial. In other words, some factors might have negative definite Ricci tensor.

It is clear from the construction that the set $U^{* *}$ is invariant under the isometry group of $M$ and is $\mathcal{L}$-complete. In summary, we have proved the following structural result for Cartan-Hadamard manifolds with degenerate Ricci.

Theorem 4. Let $M^{n}$ be a Cartan-Hadamard manifold with Ricci rank $0<r<n$. Then there exists an Iso $(M)$-invariant, $\mathcal{L}$-complete nonempty open subset $U^{* *}$ in $M^{n}$, such that the universal cover of each connected component of $U^{* *}$ is in the form

$$
Y_{1} \times \cdots \times Y_{k} \times \mathbf{R}^{q}
$$

with $k \geq 1$ and $q \geq 0$, where each $Y_{i}$ is a Riemannian manifold with nonpositive sectional curvature, foliated by its Ricci kernel foliation $\mathcal{L}_{i}$ whose leaves are totally geodesic complete flats, and its orthogonal complement $\mathcal{L}_{i}^{\perp}$ in $Y_{i}$ does not admit $R$-split. Thus when $Y_{i}$ has degenerate Ricci, either all of its conullity operators are nilpotent and the product of any two of them is zero (type $N$ ), or all of the its conullity operators are in the form $a I+b D$ with $D^{2}=-I$ (type $J$ ). 
In particular, when $M^{n}$ is real analytic, since local splitting always implies global splitting, we have the following consequence

Corollary 5. Let $M^{n}$ be an irreducible, real analytic Cartan-Hadamard manifold with Ricci rank $0<r<n$. Then either it is of type $N$, where the product of any two conullity operators is zero, or it is of type $J$, where all the conullity operators are in the form $a I+b D$ with $D^{2}=-I$.

2.2. Further restrictions. In this subsection, we will show that within each irreducible $R$-block there will be further restrictions in both the type $\mathrm{J}$ and type $\mathrm{N}$ cases. We will be working on those factors $Y_{i}$ in Theorem 4 which has degenerate Ricci. Denote by $\mathcal{L}_{i}$ its Ricci kernel foliation, and by $n_{i}, r_{i}$, and $s_{i}$ its dimension, Ricci rank, and core number, respectively.

For such a factor $Y_{i}, \mathcal{L}_{i}^{\perp}$ will not admit any $R$-split. Also, the leaves of $\mathcal{L}_{i}$ cannot vary in parallel terms, because otherwise its orthogonal complement $\mathcal{L}_{i}^{\perp}$ in $Y_{i}$ would be integrable (i.e., a foliation), which would contradict the construction of $Y_{i}$. Therefore, $Y_{i}$ will be either of type $J$ or of type $N$. In the first case, all conullity operators $C_{T}$ are in the form $a I+b D$ with $D^{2}=-I$, while in the latter case, all conullity operators are nilpotent and the product of any two of them is zero. We begin with the type $\mathrm{J}$ case.

Theorem 6. If $Y_{i}$ is a type $J$ factor in Theorem 4, then the leaves of its Ricci kernel foliation $\mathcal{L}_{i}$ are 1-dimensional, and along each leaf the trace of $C_{T}$ vanishes at exactly one point.

Proof. For simplicity, let us drop the subscript $i$ and simply write $Y^{n}, \mathcal{L}, r$ and $s$. All the conullity operators $C_{T}$ are in the form $a I+b D$ with $D^{2}=-I$. Denote by $Y^{\prime}$ the open subset in $Y$ where the kernel

$$
\mathcal{K}=\left\{T \in \mathcal{L} \mid C_{T}=0\right\}
$$

has minimum dimension (which is $s$ ). $\mathcal{K}$ becomes a distribution in $Y^{\prime}$. It is easy to see that, for any $x \in Y^{\prime}$, one can choose vectors $\left\{T_{1}, \ldots, T_{m}\right\}$ in $\mathcal{L}_{x}$ such that $C_{T_{i}}=a_{i} I+b_{i} D_{i}$, where all $b_{i}>0$, and

$$
D_{i}^{2}=-I, \quad D_{i} D_{j}+D_{j} D_{i}=0 \quad \text { when } i \neq j .
$$

Here $m=n-r-s$ is the dimension of $\mathcal{C}$, the linear span of all $C_{T}$. Note that $r$ is necessarily even here.

In this case, $\left\{I, D_{1}, \ldots, D_{m}\right\}$ generates the Clifford algebra. Thus $m$ must be less than $\rho(r)$ and the Radon-Hurwitz number defined by

$$
\rho(r)=2^{c}+8 d, \quad \text { if } r=(2 a+1) 2^{c+4 d},
$$

where $a, c, d$ are nonnegative integers with $c \leq 3$. We have $\rho(r) \leq r$, and the equality can only occur when $r=1,2,4$ or 8 . In [GZ], by a detailed check on the curvature equations for the $r=4$ and $r=8$ cases, the possibility of $m=r-1$ was ruled out. So one has $m \leq r-2$, which helped to establish the main inequality of GZ.

First, let us prove $m=1$. Note that when $r$ is not divisible by 4 , the RadonHurwitz number $\rho(r)=2$; thus $m<2$. So in the following we will assume $r=4 q$, where $q$ is a positive integer.

Assume the contrary, namely $m \geq 2$. Then there are two linear transformations $C_{T}=a I+b A$ and $C_{S}=c I+d B$ on the $r$-dimensional vector space $V=\mathcal{L}_{x}^{\perp}$ such 
that $b, d>0$ and

$$
A^{2}=B^{2}=-I, \quad A B+B A=0 .
$$

Both $A$ and $B$ will satisfy the curvature equations, since the contribution of the identity matrix in the curvature equation is zero by the first Bianchi identity. It is not hard to show that, under a suitable choice $\left\{e_{1}, \ldots, e_{r}\right\}$ of basis of $V, A$ and $B$ can be represented by the matrices (which we will still denote by $A$ and $B$ )

$$
A=J_{4 q}, \quad B=\left[\begin{array}{cc}
J_{2 q} & 0 \\
0 & -J_{2 q}
\end{array}\right], \quad \text { where } J_{2 k}=\left[\begin{array}{cc}
0 & -I_{k} \\
I_{k} & 0
\end{array}\right] .
$$

For $D=A$ or $B$, the curvature equation for $D$ says that

$$
R(X, Y) D(Z)+R(Y, Z) D(X)+R(Z, X) D(Y)=0
$$

for any $X, Y, Z \in V$. If we choose $Z=D(X)$, then $D(Z)=-X$ by the fact that $D^{2}=-I$. So the above equation implies

$$
K(X, Y)+K(D(X), Y)=-R(X, D(X), Y, D(Y))
$$

for any $X, Y \in V$. Here as before, $K(X, Y)=R(X, Y, Y, X)$. We claim the following

Claim 6. For any $1 \leq k \leq q, K\left(e_{i}, e_{j}\right)=0$ if $i$ and $j$ both belong to the set $\Lambda_{k}=\{k, k+q, k+2 q, k+3 q\}$.

For simplicity, let us denote those four indices in $\Lambda_{k}$ simply by $\{1,2,3,4\}$, and write $K_{i j}$ for $K\left(e_{i}, e_{j}\right)$. We have

$$
A\left(e_{1}\right)=-e_{3}, \quad A\left(e_{2}\right)=-e_{4}, \quad B\left(e_{1}\right)=-e_{2}, \quad B\left(e_{3}\right)=e_{4} .
$$

By $(*)$ for $D=A$, we get

$$
\begin{aligned}
& K_{12}+K_{23}=K_{14}+K_{34}=-R_{1324}, \\
& K_{12}+K_{14}=K_{23}+K_{34}=-R_{1324} .
\end{aligned}
$$

Therefore, $K_{12}=K_{34}=-\gamma, K_{14}=K_{23}=-\beta$, and $R_{1324}=\gamma+\beta$.

Similarly, by using $(*)$ for $D=B$, we get

$$
\begin{aligned}
& K_{13}+K_{23}=K_{14}+K_{24}=R_{1234}, \\
& K_{13}+K_{14}=K_{23}+K_{24}=R_{1234} .
\end{aligned}
$$

Therefore, $K_{13}=K_{24}=-\alpha$, and $R_{1234}=-\alpha-\beta$. By the first Bianchi identity, we have

$$
R_{1423}=R_{1324}-R_{1234}=\alpha+2 \beta+\gamma .
$$

We claim that the nonpositivity of the sectional curvature will now force $K_{i j}=0$ for all $1 \leq i, j \leq 4$.

To see this, consider $X=e_{1}-e_{2}$ and $Y=e_{3}+e_{4}$, and write $e_{i j}$ for $e_{i} \wedge e_{j}$. We have $X \wedge Y=e_{13}+e_{14}-e_{23}-e_{24}$. By applying the curvature equations, one could also get

$$
R_{1314}=R_{1323}=-R_{1424}=-R_{2324}
$$

So we have

$$
\begin{aligned}
0 & \leq R(X \wedge Y, X \wedge Y)=2 \alpha+2 \beta-2 R_{1324}-2 R_{1423} \\
& =2 \alpha+2 \beta-2(\beta+\gamma)-2(\alpha+2 \beta+\gamma) \\
& =-4(\beta+\gamma)
\end{aligned}
$$


Therefore, $\beta=\gamma=0$. Since $\gamma=-K_{12}=0$, by Lemma 2 of [GZ], we have

$$
\left|R_{1234}\right| \leq \frac{1}{3}(\alpha+\beta) \text {. }
$$

This together with the fact $R_{1234}=-(\alpha+\beta)$ imply that $\alpha=0$ as well. So $R_{i j c l}=0$ for all $1 \leq i, j, c, l \leq 4$ in this case. This proves Claim 6 .

Claim 7. For any $1 \leq k<l \leq q, K\left(e_{i}, e_{j}\right)=0$ if

$$
i \in\{k, k+q, k+2 q, k+3 q\}, \quad j \in\{l, l+q, l+2 q, l+3 q\} .
$$

Again for simplicity, let us denote the first four indices by $1,2,3,4$ and the next four indices by $5,6,7,8$. Consider the four indices $\{1,2,5,6\}$. Just as in the proof of Claim 1, the curvature equations of $A$ and $B$ for these four indices imply

$$
K_{15}=K_{26}=-x, \quad K_{16}=K_{25}=-y, \quad R_{1256}=x+y .
$$

Since $K_{12}=0$, by Lemma 2 of [GZ, we get

$$
x+y=\left|R_{1256}\right| \leq \frac{1}{3}(x+y) .
$$

Thus $x=y=0$. The other cases of Claim 7 can be proved similarly.

By Claim 6 and Claim 7, we know that the curvature $R$ actually vanishes, which of course violates the fact that the Ricci tensor is negative definite on $\mathcal{L}^{\perp}$. This means that there can be at most one (linearly independent) conullity operator; that is, $m=1$.

Next, we want to show that $s=0$. This together with $m=1$ would mean that the leaves of $\mathcal{L}$ are 1-dimensional. Near a generic point $x$, denote by $\mathcal{L}=\mathcal{K} \oplus \mathcal{L}^{\prime}$ the orthogonal decomposition, where $\mathcal{K}=\left\{T \in \mathcal{L} \mid C_{T}=0\right\}$. The distribution $\mathcal{L}^{\prime}$ has rank $m=1$. Let $\left\{e_{0}, e_{\alpha}, e_{i}\right\}$ be a local orthonormal tangent frame of $Y^{n}$ such that $e_{0} \in \mathcal{L}^{\prime}$ and the $e_{\alpha}$ 's are in $\mathcal{K}$. Again denote by $\theta, \Theta$ the connection and curvature matrices under the frame $e$. We have

$$
\theta_{\alpha i}=\sum_{a=1}^{n}\left\langle\nabla_{e_{a}} e_{\alpha}, e_{i}\right\rangle e_{a}=0
$$

since $C_{e_{\alpha}}=0$. Therefore

$$
\begin{aligned}
0 & =\Theta_{\alpha i}=d \theta_{\alpha i}-\sum_{a=1}^{n} \theta_{\alpha a} \wedge \theta_{a i} \\
& =-\theta_{\alpha 0} \wedge \theta_{0 i}
\end{aligned}
$$

So for any $j, k$, we have

$$
\left\langle\nabla_{e_{j}} e_{\alpha}, e_{0}\right\rangle\left\langle\nabla_{e_{k}} e_{0}, e_{i}\right\rangle=\left\langle\nabla_{e_{k}} e_{\alpha}, e_{0}\right\rangle\left\langle\nabla_{e_{j}} e_{0}, e_{i}\right\rangle .
$$

This is $A_{\alpha j} C_{k i}=A_{\alpha k} C_{j i}$, where $C=C_{e_{0}}$ and $A_{\alpha j}=\left\langle\nabla_{e_{j}} e_{\alpha}, e_{0}\right\rangle$. Since $C$ is invertible, by multiplying its inverse matrix on the right and adding it up, we get

$$
A_{\alpha j} \delta_{k l}=A_{\alpha k} \delta_{j l}
$$

for any $l$. For any $\alpha$ and any $j$, by choosing $l=k \neq j$, we get $A_{\alpha j}=0$. This means $\left\langle\nabla_{e_{j}} e_{\alpha}, e_{0}\right\rangle=0$, so $\mathcal{K}$ is a parallel distribution. This would lead to a nontrivial flat Euclidean factor of $Y^{n}$ if $s>0$. So we know that $s=0$ for any type $J$ factor.

It is not hard to see that along any leaf $L$ (which is a geodesic) of $\mathcal{L}$, the trace $\operatorname{tr}\left(C_{T}\right)$ vanishes exactly once along $L$, so we will skip it. This completes the proof of Theorem 6 . 
For a type $\mathrm{J}$ factor $Y_{i}$ in Theorem 4, the Ricci kernel foliation has 1-dimensional leaves. In the open subset $Y_{i}^{\prime} \subseteq Y_{i}$ where $C_{T}$ is not zero, each leaf contains a unique point where the trace of $C_{T}$ vanishes. We will call this geometrically distinctive point the belt point of that leaf. The set of all belt points will be denoted by $S_{i}$ and is called the belt of $Y_{i}$. It is a smooth hypersurface in $Y_{i}^{\prime}$, transversal to the foliation $\mathcal{L}_{i}$.

If we use the belt points to be the origin of the leaves of $\mathcal{L}_{i}$ and let $T$ be the unit vector in the $\mathcal{L}_{i}$ direction, then it is easy to see that in $Y_{i}^{\prime}$ there is a smooth function $\lambda>0$ and

$$
C_{T}=a I+b D, \quad a=\frac{-t}{t^{2}+\lambda^{2}}, \quad b=\frac{\lambda}{t^{2}+\lambda^{2}}
$$

where $D^{2}=-I$. Both $\lambda$ and $D$ are constant along each leaf of $\mathcal{L}_{i}$.

As an example, consider the cubic threefold in $\mathbf{R}^{4}$,

$$
M^{3}=\left\{(t, x, y, z) \mid x^{2}-y^{2}+2 t x y=z\left(1+t^{2}\right)\right\},
$$

equipped with the restriction of the Euclidean metric

$$
d s^{2}=d t^{2}+d x^{2}+d y^{2}+d z^{2} .
$$

It is an irreducible real analytic Cartan-Hadamard manifold of type J. The Ricci rank $r=2$, and the leaves of $\mathcal{L}$ are given by

$$
L_{(u, v)}=\left\{\left(t, u-t v, v+t u, u^{2}-v^{2}-2 t u v\right) \mid t \in \mathbf{R}\right\},
$$

where $(u, v) \in \mathbf{R}^{2}$. Note that, despite the fact that $M^{3}$ has many zero sectional curvatures, it is of geometric rank 1 in the sense of Ballmann, Brin and Eberlein.

This example and some others were discussed in GZ and were originated from WZ.

Note that the isometry group of $M^{3}$ is finite. This is because the origin is the unique point realizing the minimum of the scalar curvature, so any isometry of $M^{3}$ will fix the origin, and it also needs to respect the directions of $\mathcal{L}$ and the conullity operator. In particular $M^{3}$ cannot be isometric to the universal cover of a compact manifold.

Next let us discuss the type $\mathrm{N}$ factors $Y_{i}$ in Theorem 4. As before, we will denote by $Y_{i}^{\prime}$ the open subset where $\mathcal{K}$ has minimum dimension $s_{i}$. Again for the sake of simplicity, let us drop the subscript $i$ in the writing of $Y_{i}, Y_{i}^{\prime}, n_{i}, r_{i}, s_{i}$, or $\mathcal{L}_{i}$. For any $x \in Y^{\prime}$, write

$$
\mathcal{I}_{x}=\sum \operatorname{im}\left(C_{T}\right), \quad \mathcal{J}_{x}=\bigcap \operatorname{ker}\left(C_{T}\right),
$$

where $T$ is running through all directions in $\mathcal{L}_{x}$. Denote by $Y^{\prime \prime} \subset Y^{\prime}$ the open subset, where $\mathcal{I}_{x}$ has the maximum rank and $\mathcal{J}_{x}$ has the minimum rank. In $Y^{\prime \prime}$, we have distributions $\mathcal{I}$ and $\mathcal{J}$ since $C_{T} C_{S}=0$ for any $T$ and $S, \mathcal{I} \subseteq \mathcal{J}$. For convenience, let us denote by $\mathcal{N}, \overline{\mathcal{I}}$ the distributions in $Y^{\prime \prime}$ such that both

$$
\mathcal{L}^{\perp}=\mathcal{J} \oplus \overline{\mathcal{I}} \quad \text { and } \quad \mathcal{J}=\mathcal{I} \oplus \mathcal{N}
$$

are orthogonal decompositions. Let us also write $\widetilde{\mathcal{L}}=\mathcal{L} \oplus \mathcal{I}$. We prove the following

Theorem 7. Let $Y_{i}$ be a type $N$ factor as in Theorem 4. Then in the open subset $Y_{i}^{\prime \prime}$, both $\widetilde{\mathcal{L}}_{i}$ and $\mathcal{N}_{i}$ are totally geodesic foliations, and the leaves of $\widetilde{\mathcal{L}}_{i}$ are flat. In particular, the Ricci kernel foliation $\mathcal{L}_{i}$ is contained in a larger (in rank) totally geodesic foliation $\widetilde{\mathcal{L}}_{i}$ with flat leaves. Furthermore, $\nabla_{\widetilde{\mathcal{L}}_{i}} \mathcal{N}_{i} \subseteq \mathcal{N}_{i}$ and $\nabla_{\mathcal{N}_{i}} \widetilde{\mathcal{L}}_{i} \subseteq \widetilde{\mathcal{L}}_{i}$. 
Proof. Again let us drop the subscript $i$. Fix any point $x \in Y^{\prime \prime}$. For any $T \in \mathcal{L}_{x}$, by applying the curvature equation to $X, Y, Z$, where $Y \in \operatorname{ker}\left(C_{T}\right)$ and $X=C_{T}(Z)$, we know that the sectional curvature $K(X, Y)=R(X, Y, Y, X)=0$. So

$$
K_{\mathrm{im}\left(C_{T}\right), \operatorname{ker}\left(C_{T}\right)}=0
$$

for any $T$. Thus $K(\mathcal{I}, \mathcal{J})=0$. Since $\mathcal{L}$ is totally geodesic and $\mathcal{J}=\mathcal{I} \oplus \mathcal{N}$ is the common kernel of all the conullity operators, we have

$$
\nabla_{\mathcal{L}} \mathcal{L} \subseteq \mathcal{L}, \quad \nabla_{\mathcal{J}} \mathcal{L} \subseteq \mathcal{L}
$$

By using the structure equations and the properties of $\mathcal{L}, \mathcal{I}$ and $\mathcal{N}$, etc., one can show that

$$
\left\langle\nabla_{X} \mathcal{I}, \mathcal{N}\right\rangle=\left\langle\nabla_{X} \mathcal{I}, \overline{\mathcal{I}}\right\rangle=\left\langle\nabla_{X} \mathcal{N}, \overline{\mathcal{I}}\right\rangle=0
$$

for any $X$ perpendicular to $\overline{\mathcal{I}}$. We will leave the details to the readers.

Both type $\mathrm{J}$ and type $\mathrm{N}$ manifolds resemble some special kind of developable submanifolds in the Euclidean space. See [FW], W], and [WZ] for more examples and discussions. However, not all such manifolds come this way. For instance, there are examples of irreducible real analytic Cartan-Hadamard manifolds $M^{3}$ of type $\mathrm{J}$ or type $\mathrm{N}$ which are nowhere locally isometric to any hypersurface in $\mathbf{R}^{4}$ ( $\S 5$ of GZ).

Also, in the type $\mathrm{N}$ case, the core number $s$ does not have any upper bound. For instance, there are irreducible type $\mathrm{N}$ manifolds $M^{n}$ with $r=2$ and $s=n-3$ in any dimension $n \geq 3$.

One can state the combination of Theorems 4,6 , and 7 , which provides a structural description for Cartan-Hadamard manifolds with degenerate Ricci. We will skip it here. Instead, we will just state the special case when the manifold is irreducible and real analytic. In this case the picture is particularly simple:

Corollary 8. Let $M^{n}$ be an irreduicble real analytic Cartan-Hadamard manifold with Ricci rank $0<r<n$. Then one of the following two cases must occur:

1) $M^{n}$ is of type $J$. The leaves of the Ricci kernel foliation $\mathcal{L}$ are 1-dimensional. In the open dense set $U^{\prime}$, along each leaf $\gamma(t)$ of $\mathcal{L}$, the conullity operator $C_{T}=$ $a I+b D$, where $D^{2}=-I$ and $\nabla_{T} D=0$. Here $T=\gamma^{\prime}(t), b>0$. The function $a$, which is $\frac{1}{n-1}$ of the trace of $C_{T}$, vanishes exactly once on $\gamma$. So the zero locus of a, called the belt, is a real analytic hypersurface in $U^{\prime}$ transversal to $\mathcal{L}$.

2) $M^{n}$ is of type $N$. In this case $n-r-s \leq r-1$, and the product of any two conullity operators is zero. In the open dense subset $U^{\prime \prime} \subseteq M^{n}$ there are totally geodesic foliations $\widetilde{\mathcal{L}}$ and $\mathcal{N}$, where the first is formed by adding to $\mathcal{L}$ the image spaces of all $C_{T}$, while the orthogonal direct sum $\widetilde{\mathcal{L}} \oplus \mathcal{N}$ is the the sum of $\mathcal{L}$ with the common kernel space of all $C_{T}$. It holds that $\nabla_{\widetilde{\mathcal{L}}} \mathcal{N} \subseteq \mathcal{N}, \nabla_{\mathcal{N}} \widetilde{\mathcal{L}} \subseteq \widetilde{\mathcal{L}}$, and the leaves of $\widetilde{\mathcal{L}}$ are flat.

Note that the sets $U, U^{\prime}$ and $U^{\prime \prime}$ are all $\mathcal{L}$-complete and are preserved (as a set) by any isometry of $M$. The belt $S$ in the type $\mathrm{J}$ case is also preserved by any isometry of $M$.

\section{The PRoOf of TheOrem 1}

In this section, we will prove Theorem 1 stated in the Introduction, by using our detailed information on its universal cover discussed in the previous section. 
Throughout this section, we will assume that $N^{n}$ is a complete Riemannian manifold with finite volume and with nonpositive and bounded sectional curvature. We also assume that the Ricci rank of $N^{n}$ satisfies $0<r<n$. Denote by $\pi: M^{n} \rightarrow N^{n}$ the universal covering space and by $\Gamma$ the deck transformation group.

Again denote by $U \subseteq M^{n}$ the open subset where the Ricci tensor has rank $r$, and by $\mathcal{L}$ the Ricci kernel foliation in $U$. Our goal is to show that the nearby leaves of $\mathcal{L}$ are always parallel to each other, or equivalently, all the conullity operators vanish. So from now on, we will assume that not all conullity operators vanish and will try to derive a contradiction.

Write $U^{\prime} \subseteq U$ for the open (and now nonempty) subset where the linear span of the conullity operators has maximum dimension $m$. For each $x \in U^{\prime}$, denote by $k(x)$ the number of irreducible blocks of $R$-splits for $\mathcal{L}_{x}^{\perp}$ (cf. Claim 1 in $\S 2$ ). Denote by $U^{0} \subseteq U^{\prime}$ the open subset where $k(x)=k$, the minimum in $U^{\prime}$.

In the next two subsections, we will deal with the case when $k=1$; namely, when $R$ does not split at a generic point of $U^{\prime}$. The case $k>1$ will be handled in the last subsection.

3.1. The type $\mathbf{J}$ case. In this subsection, we assume $k=1$ and the open subset $U_{J} \neq \phi$, where $U_{J} \subseteq U^{0}$ consists of points where some conullity operator is nondegenerate. At each point $x \in U_{J}$, we are in the type $\mathrm{J}$ situation. We will derive a contradiction to the fact that $M^{n}$ covers a finite volume quotient.

Notice that, just like $U, U^{\prime}$ or $U^{0}$, the open subset $U_{J}$ is $\mathcal{L}$-complete and invariant under the isometries of $M^{n}$. That is, for any $x \in U_{J}$, the leaf $\mathcal{L}_{x}$ is contained in $U_{J}$; for any isometry $f$ of $M^{n}$, it holds that $f\left(U_{J}\right)=U_{J}$.

From the previous section, we know that there exists the belt $S$, which is a smooth hypersurface in $U_{J}$ that is transversal to the foliation $\mathcal{L}$ of geodesics. Clearly, for any isometry $f$ of $M^{n}$, it holds $f(S)=S$, since the conullity operators are respected by $f$ and $S$ is the zero locus of the trace of the conullity operator.

Fix a point $x_{0} \in S$. Let $B_{\epsilon}$ be the geodesic ball of radius $\epsilon$ centered at $x_{0}$. When $\epsilon>0$ is sufficiently small, $B_{\epsilon}$ is contained in $U_{J}$ and $S_{\epsilon}:=B_{\epsilon} \cap S$ is topologically a ball (of dimension $n-1$ ). We also let $2 \epsilon$ be smaller than the convexity radius at point $\pi\left(x_{0}\right) \in N^{n}$ in the quotient.

Denote by $L_{\epsilon}$ the open subset in $U_{J}$ consisting of all the leaves $\mathcal{L}_{x}$ for all $x \in S_{\epsilon}$. $L_{\epsilon}$ is diffeomorphic to the product $S_{\epsilon} \times \mathbf{R}$.

For any $f \in \Gamma$ with $f \neq i d$, it is clear that $f\left(L_{\epsilon}\right) \cap L_{\epsilon}=\phi$, as $f$ needs to preserve both $S$ and $\mathcal{L}$. Therefore $L_{\epsilon}$ injects into the quotient $N^{n}$ and thus must have finite volume. This will contradict the following lemma, thus proving Theorem 1 in the case $k=1$ and $U_{J} \neq \phi$.

Lemma 1. Under the above notation, the cylinder $L_{\epsilon}$ has infinite volume for any sufficiently small $\epsilon>0$.

Proof. Let $\left(u_{2}, \ldots, u_{n}\right)$ be a local coordinate system in a sufficiently small neighborhood $x_{0} \in W \subseteq S$. Let $e_{1}, \ldots, e_{n}$ be an orthonormal tangent frame of $M^{n}$ along $W$ such that $e_{1} \in \mathcal{L}$. Extend it to an orthonormal tangent frame in $L_{W}=\bigcup_{x \in W} L_{x}$ by parallel translation along leaves $L_{x}$ of $\mathcal{L}$.

For each $x \in W$, the geodesic $L_{x}$ will be denoted by $\gamma_{x}(t)$, such that $t$ is the arc length parameter, $\gamma_{x}(0)=x$ and $\gamma_{x}^{\prime}(0)=e_{1}(x)$. Denote by

$$
h: L_{W} \rightarrow W
$$


the projection map which sends $z$ to the belt point $L_{z} \cap W$ of the leaf $L_{z}$ of $\mathcal{L}$ passing through $z$. Define a function $x_{1}$ on $L_{W}$ by $x_{1}(z)=t$ if $z=\gamma_{x}(t)$, where $x=h(z)$. Also let $x_{i}=h^{*} u_{i}, 2 \leq i \leq n$. Then $\left(x_{1}, \ldots, x_{n}\right)$ is a local coordinate system on $L_{W}$. It is clear that for any $2 \leq i \leq n, e_{1}\left(x_{i}\right)=0$, while $e_{1}\left(x_{1}\right)=1$. Let us write $a_{i j}=e_{i}\left(x_{j}\right), 2 \leq i, j \leq n$. Then the $(n-1)$ square matrix $A=\left(a_{i j}\right)$ satisfies $e_{1}(A)=C A$, where $C$ represents the conullity operator $C_{e_{1}}$ and the volume form of $M^{n}$ is

$$
d v=\operatorname{det} A^{-1} d x_{1} \wedge d x_{2} \wedge \cdots \wedge d x_{n} .
$$

Along any leaf $\gamma(t)$ of $\mathcal{L}$, we have $C=a I+b J$, where $J^{2}=-I, J^{\prime}=0$, and

$$
a=\frac{-t}{t^{2}+\lambda^{2}}, \quad b=\frac{\lambda}{t^{2}+\lambda^{2}},
$$

where $\lambda \neq 0$. Here the derivative is with respect to $t=x_{1}$. Therefore,

$$
\left(\log \operatorname{det} A^{-1}\right)^{\prime}=-\operatorname{tr}\left(A^{\prime} A^{-1}\right)=-\operatorname{tr}(C)=-(n-1) a(t) .
$$

So $\operatorname{det} A^{-1}=c\left(t^{2}+\lambda^{2}\right)^{\frac{n-1}{2}}$ for some nowhere zero function $c$ which is constant along each $\gamma(t)$. Thus the volume of $L_{W}$ is infinity.

3.2. The type $\mathbf{N}$ case. In this subsection, we still assume $k=1$, but $U_{J}=\phi$. In other words, any point in $U^{0}$ is of type $\mathrm{N}$. We want to draw a contradiction from these assumptions and hence complete the proof of Theorem 1 in the $k=1$ case.

Unlike the type $\mathrm{J}$ case, now the dimension of the kernel foliation $\mathcal{L}$ can be larger than 1, and we don't have a geometrically invariant 'belt' transversal to $\mathcal{L}$. In fact, for any $T, S$ in $\mathcal{L}$, with $S$ parallel in each leaf, it holds that

$$
\nabla_{T} C_{S}=C_{S} C_{T}=0
$$

So all the conullity operators are constant along each leaf of $\mathcal{L}$. Thus we have to rely on something else.

Let us begin with a couple of lemmas. A subset $W \subseteq M^{n}$ is said to be precisely invariant if for any deck transformation $f \in \Gamma$, either $f(W)=W$ or $f(W) \cap W=\phi$. In this case, we will denote by $\Gamma_{W}$ the stablizing subgroup of $W$.

As before, a subset $W \subseteq U$ is said to be $\mathcal{L}$-complete if for any $x \in W$, the entire leaf $L_{x}$ of $\mathcal{L}$ through $x$ is contained in $W$.

Notice that if a subset of $M^{n}$ is preserved by any isometry of $M^{n}$, then each of its connected components will be precisely invariant.

Like the set $U^{\prime \prime}$ that we have seen before, let us denote by $U_{N} \subseteq U^{0}$ the open subset where the tangent subspaces $\mathcal{I}$ and $\mathcal{J}$ have maximum or minimum dimensions, respectively. So in $U_{N}$ we have the orthogonal decomposition

$$
T M=\mathcal{K} \oplus \mathcal{L}^{\prime} \oplus \mathcal{I} \oplus \mathcal{N} \oplus \overline{\mathcal{I}}
$$

into distributions, where the sum of the first two terms is $\mathcal{L}$ and the sum of the first three terms is $\widetilde{\mathcal{L}}$. Here $\mathcal{I}$ is the total image of all the conullity operators, while $\mathcal{I} \oplus \mathcal{N}=\mathcal{J}$ is the common kernel of the conullity operators. We also know that both $\widetilde{\mathcal{L}}$ and $\mathcal{N}$ are totally geodesic foliations, the leaves of $\widetilde{\mathcal{L}}$ are flat, and $\nabla_{\widetilde{\mathcal{L}}} \mathcal{N} \subseteq \mathcal{N}$, $\nabla_{\mathcal{N}} \widetilde{\mathcal{L}} \subseteq \widetilde{\mathcal{L}}$ 
From the construction, it is clear that $U_{N}$ is $\mathcal{L}$-complete and is preserved by any isometry of $M^{n}$. So any of its connected components will be both $\mathcal{L}$-complete and precisely invariant. We have the following

Lemma 2. For any $T \in \mathcal{L}$ with $C_{T} \neq 0$ at a point $x \in U_{N}$, if $X \in \operatorname{im}\left(C_{T}\right)$ and $Y \in \operatorname{ker}\left(C_{T}\right)$, then $R_{X Y * *}=0$. Also, the curvature tensor $R$ is constant along each leaf of $\mathcal{L}$.

Proof. For any tangent vectors $a, b, c, d$ at $x$, extend them and $T$ into vector fields so that they are all parallel in the $T$-direction; that is, $\nabla_{T}(*)=0$. The second Bianchi identity says that

$$
\left(\nabla_{T} R\right)_{a b c d}+\left(\nabla_{a} R\right)_{b T c d}+\left(\nabla_{b} R\right)_{T a c d}=0
$$

Since $R_{T * * *}=0, \nabla_{T} C_{T}=C_{T}^{2}=0$, and $\nabla_{T}(*)=0$, we have

$$
\begin{aligned}
T\left(R_{a b c d}\right) & =R_{b\left(\nabla_{a} T\right) c d}+R_{\left(\nabla_{b} T\right) a c d} \\
& =R_{\left(C_{T}(a)\right) b c d}+R_{a\left(C_{T}(b)\right) c d} \\
T\left(T R_{a b c d}\right) & =2 R_{C_{T}(a) C_{T}(b) c d} .
\end{aligned}
$$

So the third order derivative of $R_{a b c d}$ in $T$ is always zero. Now the boundedness of the sectional curvature of $M^{n}$ will force any $R_{a b c d}$ to be constant in $T$, and if $C_{T}(a)=0$, then $R_{a C_{T}(b) c d}=T R_{a b c d}=0$ for any $b, c, d$. This proves the lemma.

As a consequence, we now have $R_{X Y * *}=0$ for any $X \in \mathcal{I}$ and any $Y \in \mathcal{I} \oplus$ $\mathcal{N}$. Note that Lemma 2 is the only place (in the proof of Theorem 1) where the boundedness of the curvature is used. Hopefully this is only a technical assumption, but we do not know how to remove it at this point and time.

Lemma 3. In $U_{N}$, for any $X \in \widetilde{\mathcal{L}}$ and any $T \in \mathcal{L}$ that is parallel along the leaves of $\widetilde{\mathcal{L}}$, it holds that $\nabla_{X} C_{T}=0$.

Proof. Near a generic point $x \in U_{N}$, let us choose a piece of smooth submanifold $S$ that is transversal to $\widetilde{\mathcal{L}}$ and with complementary dimension. Choose an orthonormal tangent frame of $M^{n}$ along $S,\left\{e_{\alpha}, e_{i}, e_{\mu}, e_{i}\right\}$, such that the $e_{\alpha}$ 's are in $\mathcal{L}$, the $e_{i}$ 's are in $\mathcal{I}$, the $e_{\mu}$ 's are in $\mathcal{N}$, and the $e_{\bar{i}}$ 's are in $\overline{\mathcal{I}}$. Let us locally extend every $e_{*}$ in parallel terms along each leaf of $\widetilde{\mathcal{L}}$ through $S$; we get a local orthonormal frame near $x$ which respects the decomposition of $T M$. Note that along each leaf of $\widetilde{\mathcal{L}}$, the bundles $\mathcal{N}$ and $\overline{\mathcal{I}}$ are flat by Lemma 2 .

Write $C_{\bar{i} j}^{\alpha}=-\left\langle\nabla_{e_{\bar{i}}} e_{\alpha}, e_{j}\right\rangle$ and consider the smooth function

$$
\psi=\sum_{\alpha, \bar{i}, j, \bar{k}}\left(C_{\bar{i} j, \bar{k}}^{\alpha}\right)^{2} .
$$

Here and below we will use the subscripts after the comma to denote covariant derivatives under $e_{*}$. Clearly, $\psi$ is independent of the choice of the orthogonal frame and hence is globally defined in $U_{N}$.

Along each leaf of $\mathcal{L}$ the second order derivatives of $\psi$ is constant, and

$$
\psi_{, \beta \beta}=2 \sum\left(C_{\bar{i} j, \bar{k} \beta}^{\alpha}\right)^{2}=2 \sum\left(\sum_{l} C_{\bar{k} l}^{\beta} C_{\bar{i} j, l}^{\alpha}\right)^{2} \geq 0 .
$$

So $\psi$ is a nonnegative quadratic form along any leaf $L$ of $\mathcal{L}$. Denote by $\mathcal{L}_{0}$ the kernel of $\psi$. In the open subset $U_{N}^{\prime} \subseteq U_{N}$ where $\mathcal{L}_{0}$ has minimum dimension, it becomes a distribution. Denote by $\mathcal{L}_{1}$ the orthogonal complement of $\mathcal{L}_{0}$ in $\mathcal{L}$. 
Then along each leaf of $\mathcal{L}_{1}, \psi$ is a positive definite quadratic form and thus has a unique minimum point. So when $\mathcal{L}_{1} \neq 0$, we would have a belt $S_{1}$ transversal to the foliation $\mathcal{L}_{1}$. Any isometries of $M^{n}$ will have to preserve both the foliation $\mathcal{L}_{1}$ and this belt $S_{1}$. On the other hand, a similar argument as in the proof of Lemma 1 shows that any $\mathcal{L}_{1}$-cylinder over a small ball in $S_{1}$ will have infinite volume. (In this case the conullity operators have zero traces, so the volume form under the local coordinate system is actually constant along each leaf of $\mathcal{L}_{1}$.)

This would of course violate the fact that $M^{n}$ covers a finite volume quotient. So we must have $\mathcal{L}=\mathcal{L}_{0}$; that is, $C_{\bar{i} j, l}^{\alpha}=0$ for any indices involved. This completes the proof of Lemma 3.

Now we are ready to prove Theorem 1 in the case $k=1$ and $U_{J}=\phi$.

Near a generic point $x \in U_{N}$, let us again choose a piece of smooth submanifold $S$ that is transversal to $\widetilde{\mathcal{L}}$ and with complementary dimension, and an orthonormal tangent frame of $M^{n}$ along $S,\left\{e_{\alpha}, e_{i}, e_{\mu}, e_{\bar{i}}\right\}$, such that the $e_{\alpha}$ 's are in $\mathcal{L}$, the $e_{i}$ 's are in $\mathcal{I}$, the $e_{\mu}$ 's are in $\mathcal{N}$, and the $e_{\bar{i}}$ 's are in $\overline{\mathcal{I}}$. As in the proof of Lemma 3 , we locally extend each $e_{*}$ in parallel terms along each leaf of $\widetilde{\mathcal{L}}$ through $S$, to get a local orthonormal frame near $x$ which will respect the decomposition of $T M$. This is possible because along each leaf of $\widetilde{\mathcal{L}}$ the bundles $\mathcal{N}$ and $\overline{\mathcal{I}}$ are flat by Lemma 2 .

Again denote by $\theta, \Theta$ the matrices of connection and curvature under the frame $e$, and the indices in the corresponding ranges as before. Also write

$$
C_{\bar{i} j}^{\alpha}=-\left\langle\nabla_{e_{\bar{i}}} e_{\alpha}, e_{j}\right\rangle
$$

By Lemma 3, we have $e_{k}\left(C_{\bar{i} j}^{\alpha}\right)=0$ for any $\alpha, \bar{i}$, and any $j, k$. Since we also have $\nabla_{e_{k}} e_{*}=0$; we get

$$
\sum_{\bar{l}} \theta_{k \bar{l}, \bar{i}} C_{\bar{l} j}^{\alpha}=0
$$

for any $\alpha, \bar{i}$, and any $j, k$. Multiply the above with $C_{\bar{m} j}^{\alpha}$ and sum over $\alpha$ and $j$; we get

$$
\sum_{\bar{l}} \theta_{k \bar{l}, \bar{i}} A_{\bar{l} \bar{m}}=0,
$$

where $A_{\bar{l} \bar{m}}=\sum_{\alpha, j} C_{\bar{l} j}^{\alpha} C_{\bar{m} j}^{\alpha}$. Since $A$ is positive definite, we have $\theta_{k \bar{l}, \bar{i}}=0$ for any $k, \bar{l}$ and $\bar{i}$. Since $\theta_{k \bar{l}}$ contains only the $\varphi_{\bar{i}}$ 's, where $\varphi$ is the coframe dual to $e$, we get $\theta_{k \bar{l}}=0$.

Using this and the fact that any $\theta_{k \mu}, \theta_{k \bar{l}}$ or $\theta_{\mu \bar{l}}$ only contains the $\varphi_{\bar{i}}{ }^{\prime}$, we get from the structure equation that

$$
R_{k \bar{l} k \bar{l}}=0 \quad \forall k \forall \bar{l} .
$$

This means that the Ricci curvature in the direction of $k$ would be zero, contradicting the fact that the Ricci is negative definite in $\mathcal{L}^{\perp}$. Thus the case $k=1$ of Theorem 1 is proved.

3.3. The general case. In this subsection, we will finish the proof of Theorem 1. Our goal is to show that all conullity operators vanish, so the leaves of $\mathcal{L}$ will be parallel to each other in each connected component of $U$.

We assume that not all conullity operators vanish. As before, denote by $U^{\prime} \subseteq U$ the open subset where the linear span of the conullity operators has maximum rank $m \geq 1$, and denote by $U^{0} \subseteq U^{\prime}$ the open subset where $k(x)=k$. Here $k(x)$ is the 
number of irreducible blocks in the $R$-splitting, and $k$ is the minimum of $k(x)$ for all $x \in U^{\prime}$. We already proved the case when $k=1$ in the previous two subsections. So here we assume $k \geq 2$.

In $U^{0}$, we have the orthogonal decompositions

$$
T M=\mathcal{L} \oplus \mathcal{V}_{1} \oplus \cdots \oplus \mathcal{V}_{k}
$$

into distributions which gives the irreducible $R$-split at each point in $U^{0}$. Let $\mathcal{V}_{i}^{\infty}$ be the subspace generated by $\mathcal{V}_{i}$ for each $i$ as in $\S 2$, right after Claim 5 . Denote by $U^{00} \subseteq U^{0}$ the open subset where each $\mathcal{V}_{i}^{\infty}$ has maximum dimension. Then in $U^{00}$ we have the decomposition

$$
T M=\mathcal{L}^{0} \oplus \mathcal{V}_{1}^{\infty} \oplus \cdots \oplus \mathcal{V}_{k}^{\infty}
$$

which is now invariant under parallel transports. Thus, for each connected component $U_{a}^{00}$ of $U^{00}$, its universal cover $\widetilde{U_{a}^{00}}$ is isometric to a product

$$
\widetilde{U_{a}^{00}}=\mathbf{R}^{q} \times Y_{1 a} \times \cdots \times Y_{k a} .
$$

Here $U^{0}$ and $U^{00}$ are playing the exact same role as $U^{*}$ and $U^{* *}$ before. The only difference is that the latter two are the subsets in $U$ directly, where we didn't care about the conullity operators. In the current case, since $U^{0}$ and $U^{00}$ are subsets of $U^{\prime}$, at least one of the factors $Y_{i a}$ will have to have degenerate Ricci and thus be of either type J or type N.

Note that by their construction, it is clear that the sets $U^{0}$ and $U^{00}$ are $\mathcal{L}$ complete and are invariant under any isometry of $M^{n}$.

Let us fix a connected component $U_{a}^{00}$ of $U^{00}$. Write $W$ for its universal covering space $\widetilde{U_{a}^{00}}$, and denote by $\Gamma_{W}$ a subgroup of the isometry group of $W$ that acts freely and properly discontinuously, so that

$$
W / \Gamma_{W}=\pi\left(U_{a}^{00}\right)=U_{a}^{00} / \Gamma_{a},
$$

where $\Gamma_{a}$ is the stablizing subgroup of (the precisely invariant) $U_{a}^{00}$ in the deck transformation group $\Gamma$. $W$ is the product of $\mathbf{R}^{q}$ with $k$ factors $Y_{i a}, 1 \leq i \leq k$, and at least one of these $Y_{i a}$ will have degenerate Ricci and thus be either of type $\mathrm{J}$ or of type $\mathrm{N}$.

First let us assume that there is at least one type J factor. Denote by $Y_{1}$ the product of all the type $\mathrm{J}$ factors, and write $Y_{2}$ for the product of everything else. We have

$$
W=Y_{1} \times Y_{2} .
$$

Note that any isometry of $W$ will respect this splitting, since it needs to send type J factors to type J factors. Let $\mathcal{L}_{1}$ be the Ricci kernel foliation of $Y_{1}$ and $S_{1} \subseteq Y_{1}$ be the belt. Note that $\mathcal{L}_{1}$ and $S_{1}$ are, respectively, the product of that in the factors of $Y_{1} . S_{1}$ is a smooth submanifold in $Y_{1}$ transversal to $\mathcal{L}_{1}$ and with complementary dimension. Let $S=S_{1} \times Y_{2}$.

Fix any $x=\left(x_{1}, x_{2}\right) \in W$. For each factor of $Y_{1}$, consider a geodesic ball centered at (the component of) $x_{1}$ and with sufficiently small radius, and let $B_{1}$ be their product. Also let $B_{2}$ be a small geodesic ball in $Y_{2}$ centered at $x_{2}$.

As before, denote by $L_{1}$ or $L$ the topological cylinders consisting of leaves of $\mathcal{L}_{1}$ through $B_{1}$ or $B=B_{1} \times B_{2}$, respectively. Then $L$ is isometrically the product of $L_{1}$ and $B_{2}$, while $L_{1}$ is isometric to the product of the corresponding topological cylinders from each factor in $Y_{1}$. So by Lemma 1 , we know that $L$ would have infinite volume. 
On the other hand, for any isometry $f \in \Gamma_{W}$ which is not the identity, $f(L) \cap L=$ $\phi$, since $f(B) \cap B=\phi$ when the radii are small enough. Thus $W$ injects into $N^{n}$ and must have finite volume. This means that type $\mathrm{J}$ factors cannot appear in the splitting of $W$.

Now assume that there is no type $\mathrm{J}$ factor in $W$. Denote by $Y_{1}$ the product of all the type $\mathrm{N}$ factors and by $Y_{2}$ everything else. Again we have $W=Y_{1} \times Y_{2}$ and $\Gamma_{W}$ respect the splitting. As in the previous subsection, the fact that the quotient $N^{n}$ has finite volume and bounded curvature will force the conullity operators in $Y_{1}$ to be parallel along each leaf of $\widetilde{\mathcal{L}}_{1}$, which is the foliation obtained by adding to the Ricci kernel the total image space of the conullity operators in $Y_{1}$. This will imply that the Ricci curvature in some direction in $Y_{1}$ perpendicular to $\mathcal{L}_{1}$ to be zero, and provides the contradiction needed. Since the situation is exactly analogous to the previous subsection, we will leave the details to the readers.

In summary, we have proved that type $\mathrm{N}$ factors cannot appear in $W$ either. This completes the proof of Theorem 1.

\section{ACKNOWLEDGEMENT}

We would like to thank mathematicians J. Cao, P. Eberlein, L. Florit, X. Rong, V. Schroeder, R. Spatzier, and H. Wu for helpful discussions and for their interest and encouragement. We are indebted to Mike Davis for many hours of discussion which was vital to this work. We would also like to thank the referee of this paper, who made a number of excellent suggestions and corrections which enhanced the readability of the paper.

\section{REFERENCES}

[A] K. Abe, Applications of a Riccati type differential equation to Riemannian manifolds with totally geodesic distributions, Tôhoku Math. J., 25 (1973), 425-444. MR0350671 $(50: 3163)$

[BGS] W. Ballmann, M. Gromov and V. Schroeder, Manifold of nonpositive curvature, Progress in Mathematics, vol. 61, Basel: Birkhäuser, 1985. MR:823981 (87h:53050)

[BS] V. Bangert and V. Schroeder, Existence of flat tori in analytic manifolds of nonpositive curvature, Ann. Sci. École Normale Sup., 24 (1991), 605-634. MR1132759 (92k:53110)

[CCR] J. Cao, J. Cheeger and X. Rong, Splittings and Cr-structures for manifolds with nonpositive sectional curvature, Invent. Math., 144 (2001), 139-167. MR1821146 (2002a:53049)

[CCR1] J. Cao, J. Cheeger and X. Rong, Local splitting structures on nonpositively curved manifolds and semirigidity in dimension 3, Comm. Anal. Geom., 12 (2004), 389-415. MR2074883 (2005g:53045)

[CE] S.S. Chen and P. Eberlein, Isometry groups of simply connected manifolds of nonpositive curvature, Illinois J. Math., 24 (1980), 73-103. MR550653 (82k:53052)

[CK] S.S. Chern and N. Kuiper, Some theorems on the isometric imbedding of compact Riemannian manifolds in Euclidean space, Ann. of Math., 56 (1952), 422-430. MR0050962 $(14: 408 \mathrm{e})$

[DOZ] M. Davis, B. Okun and F. Zheng, Piecewise Euclidean structures and Eberlein's Rigidity Theorem in the singular case, Geometry and Topology, 3 (1999), 303-330. MR.1714914 (2000j:53054)

[DR] M. Dajczer and L. Rodriquez, Complete real Kähler minimal submanifolds, J. Reine Angew. Math., 419 (1991), 1-8. MR.1116914 (92g:53057)

[E1] P. Eberlein, Lattices in spaces of nonpositive curvature, Ann. Math., 111 (1980), 435-476. MR:577132 (82m:53040)

[E2] P. Eberlein, A canonical form for compact nonpositively curved manifolds whose fundamental groups have nontrivial center, Math. Ann. 260 (1982) 23-29. MR664362 (84h:53049) 
[E3] P. Eberlein, Isometry groups of simply connected manifolds of nonpositive curvature, II, Acta Math., 149 (1982), 41-69. MR674166 (83m:53055)

[E4] P. Eberlein, Euclidean de Rham factor of a lattice of nonpositive curvature, J. Diff. Geom., 18 (1983), 209-220. MR710052 (84i:53042)

[E5] P. Eberlein, Symmetry diffeomorphism groups of a manifold of nonpositive curvature, II, Indiana Math. J., 37 (1988), 735-752. MR982828 (90e:53050)

[EHS] P. Eberlein, U. Hamenstädt and V. Schroeder, Manifolds of nonpositive curvature, Differential geometry: Riemannian geometry (Los Angeles, CA, 1990), 179-227, Proc. Sympos. Pure Math., 54, Part 3, Amer. Math. Soc., Providence, RI, 1993. MR1216622 (94d:53060)

[F] D. Ferus, On the completeness of nullity foliations, Michigan Math. J., 18 (1971), 61-64. MR0279733 (43:5454)

[Fl] L. Florit, On submanifolds with nonpositive extrinsic curvature, Math. Ann., 298 (1994), 187-192. MR 1252825 (95c:53075)

[FW] G. Fischer and H. Wu, Developable complex analytic submanifolds, International J. Math., 6 (1995), 229-272. MR1316302 (95k:32012)

[FZ] L. Florit and F. Zheng, On nonpositively curved Euclidean submanifolds: Splitting results, Comm. Math. Helv., 74 (1999), 53-62. MR1677106 (99m:53100)

[FZ1] L. Florit and F. Zheng, On nonpositively curved Euclidean submanifolds: Splitting results, II, J. Reine Angew. Math., 508 (1999), 1-15. MR.1676866 (99m:53101)

[GZ] D. Guler and F. Zheng, On Ricci rank of Cartan-Hadamard manifolds, Internat. J. Math., 13 (2002), 557-578. MR.1915520 (2003d:53052)

[JY] J. Jost and S.T. Yau, Harmonic maps and rigidity theorems for spaces of nonpositive curvature, Comm. Anal. Geom., 7 (1999), 681-694. MR1714940 (2000m:53055)

[S1] V. Schroeder, Existence of immersed tori in manifolds of nonpositive curvature, J. Reine Angew. Math., 390 (1988), 32-46. MR953675 (89i:53032)

[S2] V. Schroeder, Structure of flat subspaces in low-dimensional manifolds of nonpositive curvature, Manuscripta Math., 64 (1989), 77-105. MR994382 (90c:53109)

[S3] V. Schroeder, Codimension one tori in manifolds of nonpositive curvature, Geom. Dedicata, 33 (1990), 251-263. MR.1050413 (91k:53049)

[S4] V. Schroeder, Analytic manifolds of nonpositive curvature with higher rank subspaces, Arch. Math. (Basel), 56 (1991), 81-85. MR.1082005 (92a:53060)

[S5] V. Schroeder, private communications.

[W] $\mathrm{H} . \mathrm{Wu}$, Complete developable submanifolds in real and complex Euclidean spaces, International J. Math., 6 (1995), 461-489. MR.1327160 (96e:53083)

[WZ] $\mathrm{H}$. Wu and F. Zheng, Complete developable submanifolds in complex Euclidean spaces, Comm. Anal. Geom., 10 (2002), 611-646. MR.1912259 (2003d:53101)

[WZ1] H. Wu and F. Zheng, Compact Kähler manifolds with nonpositive bisectional curvature, J. Diff. Geom., 61 (2002), 263-287. MR1972147(2004b:53128)

[WZ2] $\mathrm{H}$. Wu and F. Zheng, Kähler manifolds with slightly positive bisectional curvature, Explorations in complex and Riemannian geometry, 305-325, Contemp. Math., 332, Amer. Math. Soc., Providence, RI, 2003. MR2018347 (2005d:32043)

[Z] F. Zheng, Kodaira dimensions and hyperbolicity for nonpositively curved Kähler manifolds, Comm. Math. Helv., 77 (2002), 221-234. MR.1915039 (2003d:53058)

[Z1] F. Zheng, Ricci degeneracy in nonpositively curved manifolds, Proceedings of ICCM2001, Taipei, 441-450, International Press, Boston, 2004. MR2498000

Department of Mathematics, 202 Mathematical Sciences Building, University of MisSouri, Columbia, Missouri 65211

E-mail address: dincer@math.missouri.edu

Department of Mathematics, Ohio State University, 231 West 18th Avenue, ColumBus, Ohio 43210-1174 - and - Center of Mathematical Sciences, Zhejiang University, Hangzhou 310027, People's Republic of China

E-mail address: zheng@math.ohio-state.edu 